\title{
Partitioning Euclidean Space
}

\author{
James H. Schmerl \\ Department of Mathematics, University of Connecticut, \\ Storrs, CT 06269, USA
}

\begin{abstract}
There is a partition of Euclidean $n$-space into countably many sets none of which contains the vertices of a regular $n$-simplex.
\end{abstract}

A simple consequence of van der Waerden's theorem on arithmetic progressions is that, for any triangle $T$ and any partition of the plane $\mathbb{R}^{2}$ into finitely many pieces, there is a triangle similar to $T$ whose vertices are in one of the pieces. This result, and its proof, easily extend to higher dimensions.

For an integer $n \geq 2$ we consider Euclidean space $\mathbb{R}^{n}$ as a vector space over $\mathbb{R}$ with the Euclidean norm $\|x\|$ and the usual inner product $x \cdot y$. We call a subset $T \subseteq \mathbb{R}^{n}$ an $n$-simplex if $|T|=n+1$ and $T$ is not contained in any hyperplane. An $n$-simplex $T$ is regular if there is some positive $d \in \mathbb{R}$ such that $\|x-y\|=d$ whenever $x, y \in T$ are distinct. Equivalently, $T$ is regular iff $(x-y) \cdot(x-z)=d^{2} / 2$ whenever $x, y, z \in T$ are distinct. In particular, $T \subseteq \mathbb{R}^{2}$ is a regular 2-simplex iff $T$ is the set of vertices of an equilateral triangle, and $T \subseteq \mathbb{R}^{3}$ is a regular 3-simplex iff $T$ is the set of vertices of a regular tetrahedron. Two $n$-simplices $S, T$ are similar provided there is a function $f: S \rightarrow T$ and some $r>0$ such that $\|f(x)-f(y)\|=r\|x-y\|$ whenever $x, y \in S$.

The generalization of the aforementioned result can now be stated. If $S \subseteq \mathbb{R}^{n}$ is an $n$-simplex, then, for any partition of $\mathbb{R}^{n}$ into finitely many pieces, there is an $n$-simplex similar to $S$ which is in one of the pieces.

Complementing this result, Ceder [1] proved that $\mathbb{R}^{2}$ can be partitioned into countably many pieces none of which contains all the vertices of an equilateral triangle. This was extended to $\mathbb{R}^{3}$ by Komjáth [2] who proved, using a different technique, that $\mathbb{R}^{3}$ can be partitioned into countably many pieces none of which contains the vertices of a regular tetrahedron. In this note we refine Komjáth's technique to extend this result simultaneously in two ways: to arbitrary dimension 
$n$ and to arbitrary $n$-simplices. We fix throughout an integer $n \geq 2$ which is to be the dimension of the space being considered.

Theorem. Let $S \subseteq \mathbb{R}^{n}$ be any n-simplex. There is a partition of $\mathbb{R}^{n}$ into countably many pieces none of which contains an n-simplex similar to $S$.

The proof of the theorem uses the Axiom of Choice. Some use of the Axiom of Choice is necessary since, as Steinhaus first noted, for any $n$-simplex $S \subseteq \mathbb{R}^{n}$, any $X \subseteq \mathbb{R}^{n}$ having positive Lebesgue measure contains an $n$-simplex similar to $S$. As a consequence we get that, for every $n$-simplex $S \subseteq \mathbb{R}^{n}$ and every partition of $\mathbb{R}^{n}$ into countably many measurable sets, one of the sets contains an $n$-simplex similar to $S$.

We identify the smallest infinite ordinal $\omega$ with the set $\{0,1,2, \ldots\}$ of natural numbers, and any natural number $m$ we identify with the set $\{0,1,2, \ldots, m-1\}$ of smaller natural numbers. Let us fix some $n$-simplex $S=\left\{x_{0}, x_{1}, \ldots, x_{n}\right\} \subseteq \mathbb{R}^{n}$. If $X \subseteq \mathbb{R}^{n}$ and $\psi: X \rightarrow \omega$, then we say that $\psi$ is proper if there is no $n$-simplex $T \subseteq X$ which is similar to $S$ such that $|\{\psi(x): x \in T\}|=1$. The following is an obvious reformulation of the theorem.

For every infinite cardinal $\kappa \leq 2^{\aleph_{0}}=|\mathbb{R}|$ and every real-closed field $F \subseteq \mathbb{R}$ such that $|F|=\kappa$, there is a proper $\psi: F^{n} \rightarrow \omega$.

To prove the theorem we prove (*) by induction on the cardinal $\kappa$. For $\kappa=\aleph_{0}$, the result is trivial: just let $\psi: F^{n} \rightarrow \omega$ be any one-one function.

To proceed by induction, assume that $\kappa_{0}<\kappa \leq 2^{\kappa_{0}}$ and that whenever $K \subseteq \mathbb{R}$ is a real-closed field and $|K|<\kappa$, then there is a proper function $\theta: K^{n} \rightarrow \omega$. Let $F \subseteq \mathbb{R}$ be real-closed such that $|F|=\kappa$. We easily obtain a sequence $\left\langle F_{\alpha}: \alpha<\kappa\right\rangle$ such that the following four conditions hold:

(1) $S \subseteq F_{0}^{n}$;

(2) if $\alpha<\beta<\kappa$, then $F_{\alpha}$ and $F_{\beta}$ are real-closed fields, $F_{\alpha} \subseteq F_{\beta} \subseteq F$ and $\left|F_{\beta}\right|<\kappa$;

(3) if $\beta<\kappa$ is a limit ordinal, then $F_{\beta}=\bigcup_{\alpha<\beta} F_{\alpha}$;

(4) $F=\bigcup_{\alpha<x} F_{\alpha}$.

We construct a sequence $\left\langle\psi_{\alpha}: \alpha<\kappa\right\rangle$ of proper functions $\psi_{\alpha}: F_{\alpha}^{n} \rightarrow \omega$ such that whenever $\alpha<\beta<\kappa$, then $\psi_{\alpha}=\psi_{\beta} \mid F_{\alpha}^{n}$. Clearly, then $\psi=\bigcup_{\alpha<\kappa} \psi_{\alpha}$ will be a proper function $\psi: F^{n} \rightarrow \omega$.

The sequence $\left\langle\psi_{\alpha}: \alpha\langle\kappa\rangle\right.$ is constructed by transfinite recursion. To that end, assume that $\gamma<\kappa$ and that we already have $\left\langle\psi_{\alpha}: \alpha<\gamma\right\rangle$. We wish to construct $\psi_{\gamma}$ : If $\gamma=0$, then let $\psi_{0}: F_{0}^{n} \rightarrow \omega$ be any proper function (the existence of which is guaranteed by the inductive hypothesis on $\kappa$ ). For $\gamma$ a limit ordinal, we have no choice but to set $\psi_{y}=\bigcup_{\alpha<\gamma} \psi_{\alpha}$. It remains to consider the case that $\gamma=\alpha+1$.

We need two functions to define $\psi_{\gamma}$. The first can be any proper function $\theta: F_{\gamma}^{n} \rightarrow \omega$, the existence of which is guaranteed by the inductive hypothesis on $\kappa$. The second is a function $\varphi$ as described in the next lemma. 
Lemma 1. There is $m \in \omega$ and a function $\varphi: F_{\gamma}^{n} \backslash F_{\alpha}^{n} \rightarrow m$ with the following property: whenever $T \subseteq F_{\gamma}^{n}$ is an $n$-simplex similar to $S$ such that $T \cap F_{\alpha}^{n} \neq \varnothing \neq T \backslash F_{\alpha}^{n}$, then there are $x, y \in T \backslash F_{\alpha}^{n}$ such that $\varphi(x) \neq \varphi(y)$.

Before proving Lemma 1, we show how to obtain $\psi_{\gamma}$. For $x \in F_{\gamma}^{n}$, let

$$
\psi_{\gamma}(x)= \begin{cases}\psi_{\alpha}(x) & \text { if } \quad x \in F_{\alpha}^{n}, \\ m \theta(x)+\varphi(x) & \text { if } x \notin F_{\alpha}^{n} .\end{cases}
$$

It should be clear that $\psi_{\gamma}: F_{\gamma}^{n} \rightarrow \omega$ is proper and that $\psi_{\alpha}=\psi_{y} \mid F_{\alpha}^{n}$.

Several more lemmas are needed to prove Lemma 1 . Let $S^{n-1}=$ $\left\{x \in \mathbb{R}^{n}:\|x\|=1\right\}$ be the unit $(n-1)$-sphere. The next lemma is an immediate consequence of the compactness of $S^{n-1}$.

Lemma 2. For every $\varepsilon>0$ there is $m \in \omega$ and a function $\chi: S^{n-1} \rightarrow m$ such that whenever $x, y \in S^{n-1}$ and $\chi(x)=\chi(y)$, then $x \cdot y \geq 1-\varepsilon$.

The next lemma is suggested by [2], and the proof presented here is also influenced by [2]. Recall that we have a fixed $n$-simplex $S=\left\{x_{0}, x_{1}, \ldots, x_{n}\right\}$.

Lemma 3. There exists $\varepsilon_{0}>0$ such that whenever $T=\left\{y_{0}, y_{1}, \ldots, y_{n}\right\} \subseteq \mathbb{R}^{n}$ is an n-simplex similar to $S$ for which

$$
\begin{aligned}
x_{i}=y_{i} & \text { for some } i \leq n, \\
x_{i} \neq y_{i} & \text { for some } i \leq n \\
\left\|x_{i}-y_{i}\right\|<\varepsilon_{0} & \text { for all } i \leq n
\end{aligned}
$$

then there are (necessarily distinct) $i, j \leq n$ such that $x_{i} \neq y_{i}, x_{j} \neq y_{j}$, and

$$
\frac{y_{i}-x_{i}}{\left\|y_{i}-x_{i}\right\|} \cdot \frac{y_{j}-x_{j}}{\left\|y_{j}-x_{j}\right\|}<1-\varepsilon_{0}
$$

Proof. Intending to arrive at a contradiction, assume that there is no such $\varepsilon_{0}$. Then we can assume, without loss of generality, that $x_{0}=0$ and for each integer $m \geq 1$ there is an $n$-simplex $\left\{y_{0 m}, y_{1 m}, \ldots, y_{n m}\right\}$ and $r_{m}>0$ such that, for all $i, j \leq n$,

$$
\begin{aligned}
y_{0 m} & =0, \\
y_{1 m} & \neq x_{1}, \\
\left\|y_{i m}-x_{i}\right\| & <\frac{1}{m}, \\
\left\|y_{i m}-y_{j m}\right\| & =r_{m}\left\|x_{i}-x_{j}\right\|,
\end{aligned}
$$


and

$$
\left(y_{i m}-x_{i}\right) \cdot\left(y_{j m}-x_{j}\right) \geq\left\|y_{i m}-x_{i}\right\|\left\|y_{j m}-x_{j}\right\|\left(1-\frac{1}{m}\right)
$$

Moreover, without loss of generality we can assume that $\left\|y_{1 m}-x_{1}\right\| \geq\left\|y_{i m}-x_{i}\right\|$ for all $i \leq n$.

For each $m \geq 1$, let $d_{m}=\left\|y_{1 m}-x_{1}\right\|$ and $v_{i m}=\left(1 / d_{m}\right) /\left(y_{i m}-x_{i}\right)$. Observe that $\left\|v_{i m}\right\| \leq\left\|v_{1 m}\right\|=1$ and $\lim _{m \rightarrow \infty} d_{m}=0$. Thus, without loss of generality, we can assume that the limits $v_{i}=\lim _{m \rightarrow \infty} v_{i m}$ exist, and that $v_{i}=b_{i} v_{1}$ where $0 \leq b_{i} \leq 1$. Obviously, $\left\|v_{1}\right\|=1$.

For $i, j \leq n$, let $a_{i}=\left\|x_{i}\right\|$ and $c_{i j}=x_{i} \cdot x_{j}$. Clearly, for $m \geq 1$ and $i \neq j$ we have that

$$
r_{m}^{2} a_{i}^{2}=\left\|y_{i m}\right\|^{2}=\left\|x_{i}+d_{m} v_{i m}\right\|^{2}=a_{i}^{2}+2 d_{m} x_{i} \cdot v_{i m}+d_{m}^{2}\left\|v_{i m}\right\|^{2}
$$

and

$$
\begin{aligned}
r_{m}^{2} c_{i j} & =y_{i m} \cdot y_{j m}=\left(x_{i}+d_{m} v_{i m}\right) \cdot\left(x_{j}+d_{m} v_{j m}\right) \\
& =c_{i j}+d_{m} x_{i} \cdot v_{j m}+d_{m} x_{j} \cdot v_{i m}+d_{m}^{2} v_{i m} \cdot v_{j m}
\end{aligned}
$$

From (1) we get

$$
r_{m}^{2} a_{i}^{2} a_{1}^{2}=a_{1}^{2}\left(a_{i}^{2}+2 d_{m} x_{i} \cdot v_{i m}+d_{m}^{2}\left\|v_{i m}\right\|^{2}\right)=a_{i}^{2}\left(a_{1}^{2}+2 d_{m} x_{1} \cdot v_{1 m}+d_{m}^{2}\left\|v_{1 m}\right\|^{2}\right),
$$

from which we get

$$
a_{1}^{2} x_{i} \cdot v_{i m}-a_{i}^{2} x_{1} \cdot v_{1 m}=\frac{a_{i}^{2}}{2} d_{m}\left\|v_{1 m}\right\|^{2}-\frac{a_{1}^{2}}{2} d_{m}\left\|v_{i m}\right\|^{2}
$$

Taking the limit as $m \rightarrow \infty$, we see that the right-hand side approaches 0 , so we get

$$
a_{i}^{2} x_{1} \cdot v_{1}=a_{1}^{2} x_{i} \cdot v_{i}
$$

Similarly, from (1) and (2) we get

$$
\begin{aligned}
r_{m}^{2} c_{i 1} a_{1}^{2} & =c_{i 1}\left(a_{1}^{2}+2 d_{m} x_{1} \cdot v_{1 m}+d_{m}^{2}\left\|v_{1 m}\right\|^{2}\right) \\
& =a_{1}^{2}\left(c_{i 1}+d_{m} x_{i} \cdot v_{1 m}+d_{m} x_{1} \cdot v_{i m}+d_{m}^{2} v_{i m} \cdot v_{1 m}\right),
\end{aligned}
$$

from which we get

$$
2 c_{i 1}\left(x_{1} \cdot v_{1 m}\right)-a_{1}^{2}\left(x_{i} \cdot v_{1 m}+x_{1} \cdot v_{i m}\right)=a_{1}^{2} d_{m} v_{i m} \cdot v_{1 m}-c_{i 1} d_{m}\left\|v_{1 m}\right\|^{2}
$$


Taking the limit as $m \rightarrow \infty$, we again see that the right-hand side approaches 0 , so we get

$$
a_{1}^{2}\left(x_{i} \cdot v_{1}+x_{1} \cdot v_{i}\right)=2 c_{i 1}\left(x_{1} \cdot v_{1}\right) .
$$

Replacing $v_{i}$ by $b_{i} v_{1}$ in (3) and (4) yields respectively

$$
a_{i}^{2} x_{1} \cdot v_{1}=\left(a_{1}^{2} x_{i}\right) \cdot\left(b_{i} v_{1}\right)
$$

and

$$
a_{1}^{2} x_{i} \cdot v_{1}=2 c_{1,} x_{1} \cdot v_{1}-a_{1}^{2} x_{1} \cdot b_{i} v_{1} .
$$

Therefore, plugging (6) into the right-hand side of (5) yields

$$
a_{i}^{2} x_{1} \cdot v_{1}=b_{i}\left(2 c_{1 i} x_{1} \cdot v_{1}-a_{1}^{2} b_{i} x_{1} \cdot v_{1}\right)
$$

or, equivalently,

$$
\left(x_{1} \cdot v_{1}\right)\left(a_{1}^{2} b_{i}^{2}-2 c_{1 i} b_{i}+a_{i}^{2}\right)=0 .
$$

We wish to conclude that $x_{1} \cdot v_{1}=0$. If not, then $b_{i}$ satisfies the equation

$$
a_{1}^{2} x^{2}-2 c_{1 i} x+a_{i}^{2}=0,
$$

so that $4 c_{1 i}^{2}-4 a_{1}^{2} a_{i}^{2} \geq 0$. However, since $x_{0}, x_{1}, x_{i}$ are not collinear, the Cauchy-Schwarz inequality implies $c_{1 i}^{2}<a_{1}^{2} a_{i}^{2}$, a contradiction. Thus, we have $x_{1} \cdot v_{1}=0$.

Now from (6) we get that $x_{i} \cdot v_{1}=0$ for all $i$. Since $\left\{x_{1}, x_{2}, \ldots, x_{n}\right\}$ spans $\mathbb{R}^{n}$, this implies $v_{1}=0$, contradicting that $\left\|v_{1}\right\|=1$.

If $X \subseteq \mathbb{R}^{n}$ and $x \in \mathbb{R}^{n}$, we say that $x$ is algebraic over $X$ if each coordinate of $x$ is algebraic over the set of all coordinates of elements in $X$.

Lemma 4. Let $Y, Z \subseteq \mathbb{R}^{n}$ be finite sets such that $S \subseteq Z$ and no $x \in Y$ is algebraic over $Z$. Let $\varepsilon>0$. Then there is a function $g: Y \cup Z \rightarrow \mathbb{R}^{n}$ such that whenever $T \subseteq Y \cup Z$ is an $n$-simplex similar to $S$, then so is $g[T]$; if $x \in Y$, then $0<\|x-g(x)\|<\varepsilon$; and if $x \in Z$, then $g(x)=x$.

Proof. This is just a special case of the following well known and easy consequence of Tarski's elimination of quantifiers for the first-order theory of $\mathbb{R}$ considered as an ordered field: If $m \in \omega, F \subseteq \mathbb{R}$ is a real-closed field, $X \subseteq \mathbb{R}^{m}$ is first-order definable using parameters in $F,\left\langle a_{0}, a_{1}, \ldots, a_{m-1}\right\rangle \in X$, and $\varepsilon>0$, then there is $\left\langle b_{0}, b_{1}, \ldots, b_{m-1}\right\rangle \in X$ such that, for each $i<d,\left|b_{i}-a_{i}\right|<\varepsilon$ and if $b_{i}=a_{i}$, then $a_{i} \in F$. 
We now finish the proof of the theorem by proving Lemma 1 . Let $m$ and $\chi$ be as in Lemma 2, using $\varepsilon=\varepsilon_{0}$ from Lemma 3. By compactness, it suffices to show that for each finite $X \subseteq F_{\gamma}^{n}$ there is a function $\varphi_{0}: X \backslash F_{\alpha}^{n} \rightarrow m$ with the following property: whenever $T \subseteq X$ is an $n$-simplex similar to $S$ such that $T \cap F_{\alpha}^{n} \neq \varnothing \neq T \backslash F_{\alpha}^{n}$, then there are $x, y \in T \backslash F_{\alpha}^{n}$ such that $\varphi_{0}(x) \neq \varphi_{0}(y)$.

Consider some finite $X \subseteq F_{\gamma}^{n}$ such that $S \subseteq X$, and let $Y=X \backslash F_{\alpha}^{n}$ and $Z=X \cap F_{\alpha}^{n}$. Let $r>0$ be small enough so that if $y_{1}, y_{2} \in X$ are distinct and $0 \leq i<j \leq n$, then, $\left\|y_{1}-y_{2}\right\| \geq r\left\|x_{i}-x_{j}\right\|$ and let $\varepsilon=r \varepsilon_{0}$. Now apply Lemma 4 to get $g: X \rightarrow \mathbb{R}^{n}$ satisfying the properties specified in that lemma. For each $x \in Y$, let

$$
\varphi_{0}(x)=\chi\left(\frac{x-g(x)}{\|x-g(x)\|}\right) .
$$

To see that $\varphi_{0}$ works, consider some $n$-simplex $T \subseteq X$ which is similar to $S$ such that $T \cap Z \neq \varnothing \neq T \cap Y$. Clearly, by Lemma 3, there are $x, y \in T \cap Y$ such that

$$
\frac{x-g(x)}{\|x-g(x)\|} \cdot \frac{y-g(y)}{\|y-g(y)\|}<1-\varepsilon_{0}
$$

hence, by Lemma $2, \varphi_{0}(x) \neq \varphi_{0}(y)$.

This completes the proof of Lemma 1, and, consequently, also of the theorem.

\section{References}

1. Ceder, J., Finite subsets and countable decompositions of Euclidean spaces, Rev. Roumaine Math. Pures Appl. 14 (1969), 1247-1251.

2. Komjáth, P., Tetrahedron free decomposition of $\mathbb{R}^{3}$, Bull. London Math. Soc. 23 (1991), 116-120.

Received March 9, 1992. 